\title{
Photodegradation of Basic Dyes using Nanocomposite (Silver Zinc Oxide- Copper Oxide) and Kinetic Studies
}

\author{
NASRIN KHORSHIDI ${ }^{1}$, SAIED ABEDINI KHORRAMI ${ }^{1}$, \\ MOHAMMAD EBRAHIM OLYA ${ }^{\star 2}$ and FERESHTEH MOTTIEE ${ }^{1}$ \\ ${ }^{1}$ Department of Chemistry, Faculty of Sciences, North Tehran Branch, \\ Islamic Azad University, Tehran, Iran. \\ ${ }^{2}$ Department of Environmental Research, Institute for Color Science and Technology, Tehran, Iran. \\ http://dx.doi.org/10.13005/ojc/320247 \\ (Received: June 06, 2015; Accepted: August 04, 2015)

\begin{abstract}
In this paper, $\mathrm{Ag} / \mathrm{ZnO} / \mathrm{CuO}$ nanocomposite $(\mathrm{AZCN})$ synthesized and its dye degradation ability in the presence of UV irradiation from colored wastewater was studied. The characteristics of AZCN were investigated using Fourier transform infrared (FTIR), scanning electron microscope (SEM) and X-ray diffraction (XRD). The photocatalytic activity was evaluated by photodegradation of Basic Red 18 (BR18) and Basic Violet 16 (BV16) as a model contaminant. The effects of AZCN dosage, initial dye concentration and salt on dye degradation were investigated. Kinetics analysis indicated that the dye degradation rates could be approximated at pseudo-first order model. The results indicated that $A Z C N$ as a photocatalyst could be used to degrade dyes from wastewater.
\end{abstract}

Keywords: Photocatalytic degradation, Nanocomposite, Basic dye, AOPs, Kinetic modeling.

\section{INTRODUCTION}

Adsorption, biological, filtration, coagulation and ozonation were used as common methods to treat wastewater. Each method has its advantages and disadvantages. For example, adsorption is nondestructive, since it just transfers dyes from water to another phase, thus causing secondary pollution $^{1,2}$. Consequently, regeneration of the adsorbent materials and post-treatment of solid wastes, which are expensive operations, are needed ${ }^{3,4}$. Due to the large degree of aromatics present in dye molecules and the stability of modern dyes, conventional biological treatment methods are ineffective for decolorization and degradation ${ }^{5,6}$.

Photocatalytic activity of semiconductors is well known for decomposition of pollutants in gas and liquid phases 7,8 . The process is begun by ultra violet or visible light irradiation on the semiconductor to excite an electron from the valence band to the conduction band that resulting to formation a high energy electron/hole pair. This pair may directly (or indirectly by generate the radicals) decompose organic compounds (Advanced Oxidation Processes, AOPs $)^{9,}{ }^{10}$. The advanced oxidation processes 
(AOPs) is an effective process at the degradation of air, water and wastewater pollutants, especially refractory organic substances ${ }^{11,5}$. AOPs degrades pollutants using strong oxidants such as hydroxyl radicals ( $\left.\mathrm{HO}^{*}\right)$. The advantages of AOPs over competing processes are: (1) complete degradation, (2) no waste disposal problem (3) low cost and (4) only mild temperature and pressure conditions are necessary. The mechanism of heterogeneous photocatalytic degradation of dyes was investigated in detail ${ }^{12,13}$

In this paper, AZCN was synthesized, characterized and its photocatalytic dye degradation ability from colored wastewater was investigated in details. Basic Red 18 (BR18) and Basic Violet 16 (BV16) were used as model dyes. The characteristics of AZCN were investigated using Fourier transform infrared (FTIR), scanning electron microscope (SEM) and X-ray diffraction (XRD). Photocatalytic dye degradation was studied by UVVis spectrophotometer. The effect of AZCN dosage, initial dye concentration and salt on dye degradation was evaluated. Photocatalytic dye degradation using AZCN kinetic (zero-order, first-order and secondorder) was studied.

\section{MATERIAL AND METHODS}

\section{Chemicals}

Basic Red 18 (BR18) and Basic Violet 16 (BV16) were obtained from Ciba and used without further purification. Fig. 1 shows the chemical structure of dyes. Other chemicals were obtained from Merck.

\section{Synthesize of AZCN nanocomposite Synthesize of zinc oxide/copper oxide}

$\mathrm{ZnCl}_{2}(0.2726 \mathrm{~g}), \mathrm{CuSO}_{4} \cdot 5 \mathrm{H}_{2} \mathrm{O}(0.2497 \mathrm{~g})$ and $\mathrm{NaOH}(0.4 \mathrm{~g})$ were dissolved in distilled water $(30 \mathrm{~mL})$. This solution was sealed and left static at 80 E\% $\mathrm{C}$ for $24 \mathrm{~h}$, and then cooled to room temperature. The final precipitate was separated by centrifuge, washed with distilled water and absolute alcohol several time to removal the possible residues and then dried at $80^{\circ} \mathrm{C}$ for $12 \mathrm{~h}(14)$.

\section{Synthesize of AZCN}

$0.5 \mathrm{~g}$ silver nitrate was added to $50 \mathrm{~mL}$ water, heated and stirred. Then $5 \mathrm{~g}$ zinc oxide/copper oxide nanocomposite was added, agitated and heated at $120^{\circ} \mathrm{C}$ for $1 \mathrm{~h}$. the product was calcined at $400^{\circ} \mathrm{C}$ for $4 \mathrm{~h}$.

\section{Characterization}

The functional groups of the material were studied using Fourier transform infrared (FTIR) spectroscopy (Perkin-Elmer Spectrophotometer Spectrum One) in the range of $4000-450 \mathrm{~cm}^{-1}$. The morphological structure of the material was examined by scanning electron microscopy (SEM) using a LEO 1455VP scanning microscope. Crystallization behavior of nanocomposite was identified by XRD model Siemens D-5000 diffractometer with $\mathrm{Cu} \mathrm{K} \mathrm{K}_{\alpha}$ radiation $\left(\lambda=1.5406 A^{\circ}\right)$ at room temperature.

\section{Reactor and Photocatalytic dye degradation}

Experiments were carried out in a batch mode photoreactor. The irradiation source was a UV-C lamp (200-280 nm, 9W, Philips), which was placed in the inner quartz tube of the reactor.

The photocatalytic dye degradation experiments were conducted by mixing various amounts of $A Z C N$ in photoreactor containing 800 $\mathrm{mL}$ of a dye solution (10 $\mathrm{mg} / \mathrm{L}$ ) at room temperature $\left(25^{\circ} \mathrm{C}\right)$. The solution samples were withdrawn from the reaction medium at regular time intervals. The AZCN was separated from solution and the change on the absorbance at maximum wavelength $\left(\lambda_{\max }\right)$ of dyes (489 $\mathrm{nm}$ for BR18 and $547 \mathrm{~nm}$ for BV16) was monitored by UV-vis spectrophotometer (PerkinElmer Lambda 25).

The effect of AZCN dosage on the photocatalytic dye degradation was investigated by contacting $800 \mathrm{~mL}$ of dye solution (10 mg/L) at room temperature $\left(25^{\circ} \mathrm{C}\right)$ for $4 \mathrm{~h}$. Different amounts of $\mathrm{AZCN}$ were applied.

The effect of initial dye concentration on the photocatalytic dye degradation was studied. The AZCN (0.05 g for BV16 and $0.1 \mathrm{~g}$ for BR18) was added to $800 \mathrm{~mL}$ of different dye concentrations (10, 20, 30 and $40 \mathrm{mg} / \mathrm{L})$.

The effect of salt on the photocatalytic dye degradation was studied. Different salts $\left(\mathrm{Na}_{2} \mathrm{SO}_{4}\right.$, $\mathrm{NaCl}$ and $\left.\mathrm{NaHCO}_{3}\right)(0.02 \mathrm{~mol})$ were added to 800 $\mathrm{mL}$ of dye solution (10 mg/L) containing AZCN (0.05 
$\mathrm{g}$ for BV16 and $0.1 \mathrm{~g}$ for BR18) at room temperature $\left(25^{\circ} \mathrm{C}\right)$.

\section{RESULTS AND DISCUSSION}

\section{Characterization}

The FT-IR spectrum of AZCNnanocomposite was shown in Fig. 2. It has two peaks at $3450 \mathrm{~cm}^{-1}$ and $500-600 \mathrm{~cm}^{-1}$ which indicate $\mathrm{O}-\mathrm{H}$ stretching vibration and metal-oxygen vibration, respectively. The peak at $1625 \mathrm{~cm}^{-1}$ was attributed to $\mathrm{OH}$ bending of molecular water ${ }^{15}$.

SEM has been a primary tool for characterizing the surface morphology and fundamental physical properties of material surface. It is used to determine the particle shape and appropriate size distribution of the material. The SEM micrograph of the AZCN (Fig. 3) shows a relatively homogeneous particle size distribution of $A Z C N$
Fig. 4 illustrates the X-ray diffraction (XRD) pattern of the zinc oxide/copper oxide nanocomposite. Diffraction peaks are in good agreement with those of the standard patterns of hexagonal wurtzite<smiles>CCN(CC[N+](C)(C)Cl)c1ccc(N=Nc2ccc([N+](=O)[O-])cc2Cl)cc1</smiles>

(a)<smiles></smiles>

Fig. 1: The chemical structure of dyes (a) BR18 and (b) BV16

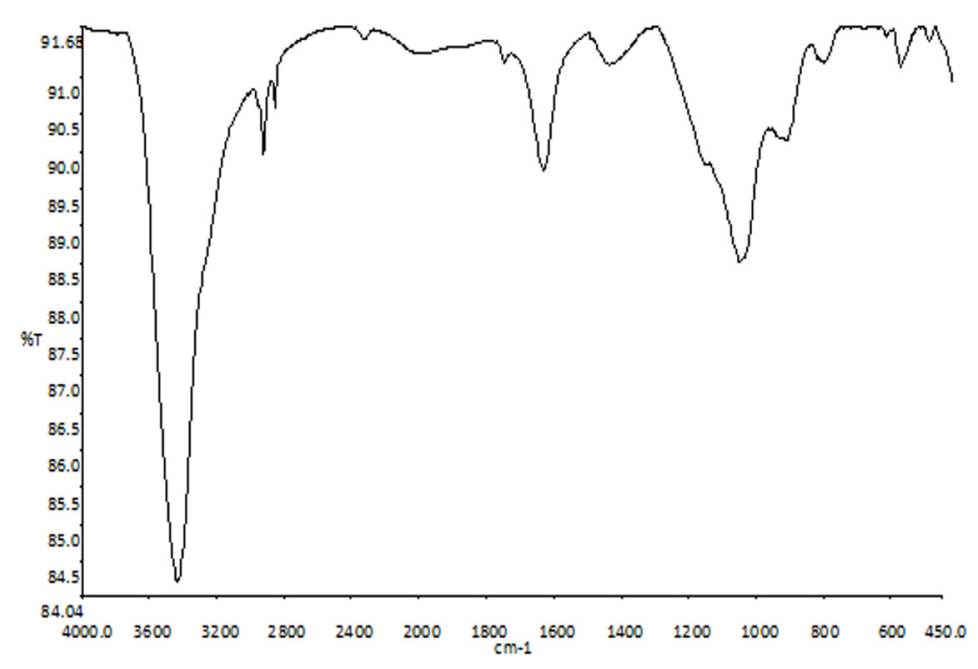

Fig. 2: FTIR spectrum of AZCN

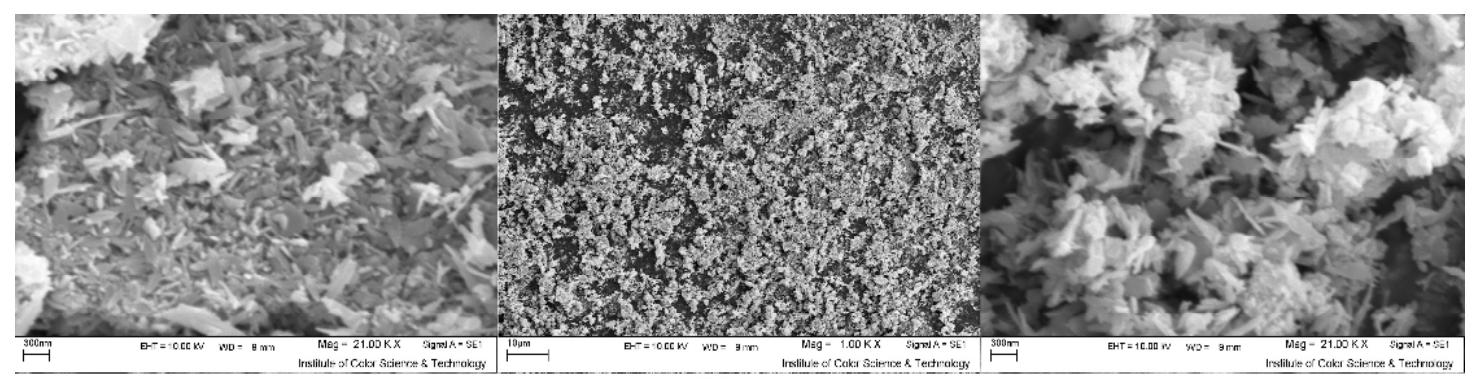

(a)

(b)

(c)

Fig. 3: SEM image of AZCN 
ZnO (JCPDS Card No. 36-1451) and monoclinic CuO (JCPDS Card No. 05-0661). In Fig. 4, there are two sets of diffraction peaks for the zinc oxide/ copper oxide nanocomposite sample, which are correspondingly ascribed to hexagonal wurtzite $\mathrm{ZnO}$ and monoclinic $\mathrm{CuO}$, and no peaks of other phases and impurities were detected ${ }^{14}$.

\section{Degradation of dyes}

The UV-Vis spectra of the dyes at $200 \mathrm{~nm}$ $\leq \lambda \leq 650 \mathrm{~nm}$ during the degradation process using UV/ AZCN at different time intervals were studied (Fig. 5). The result showed that the maximum absorbance in visible region of spectra decreases because chromophore of dyes is the most active sites for degradation attack.

\section{Effect of operational parameters on dye degradation \\ AZCN dosage}

Fig. 6 shows the effect of AZCN dosage on the photocatalytic dye degradation. The photodegradation in the absence of $A Z C N$ is $2 \%$ and $1.5 \%$ for BR18 and BV16, respectively. Considering that the UV alone is not effective to oxidize dyes in the colored wastewater samples, it needs to be activated by a catalyst. It is observed that the simultaneous use of AZCN with UV to a certain extent yielded a significant improvement of dye degradation compared to that of the UV degradation alone (Fig. 6).

AZCN is an effective photocatalyst by utilizing light energy to create $\mathrm{e}^{-/ \mathrm{h}^{+}}$pairs on its

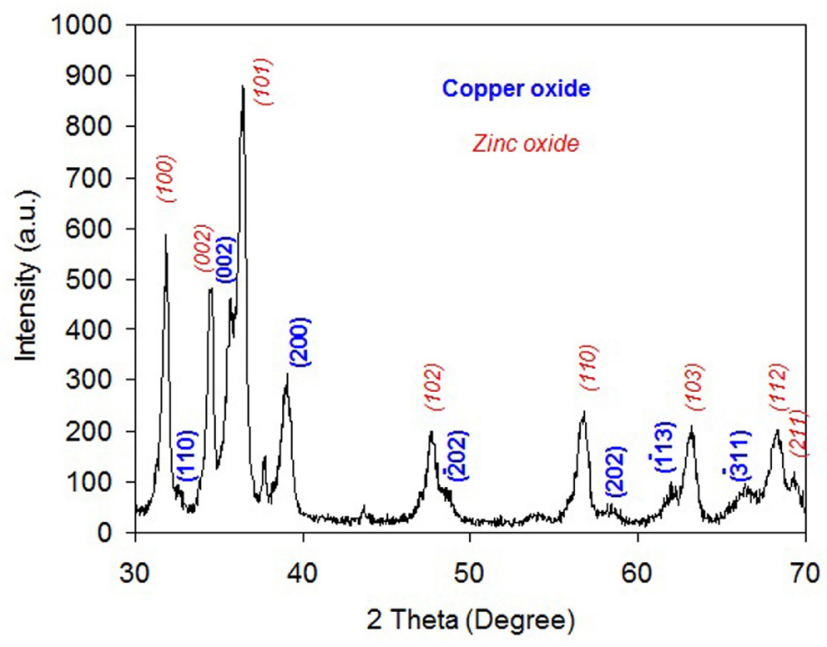

Fig. 4: XRD pattern of zinc oxide/copper oxide nanocomposite

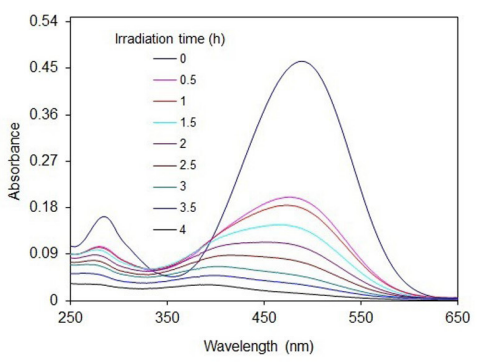

(a)

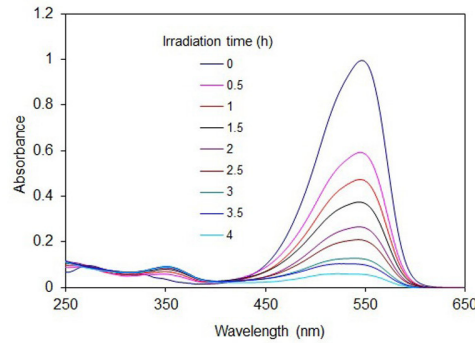

(b)

Fig. 5: UV-Vis spectrum of dyes during the degradation process using UV/AZCN (a) BR18 and (b) BV16 


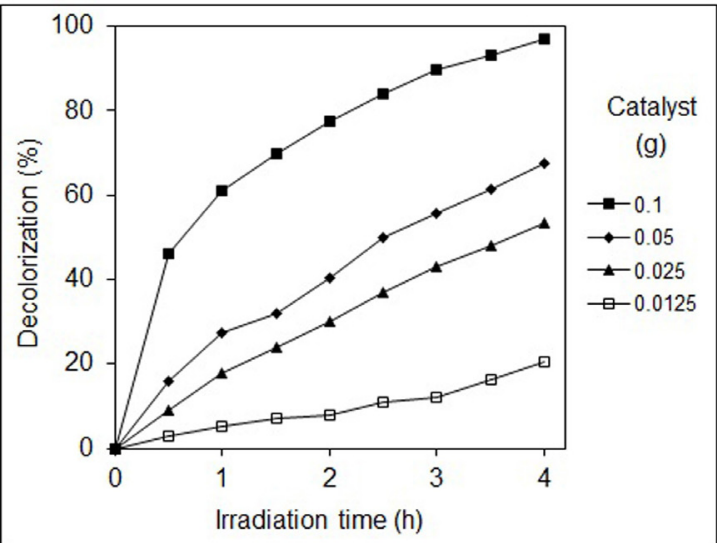

(a)

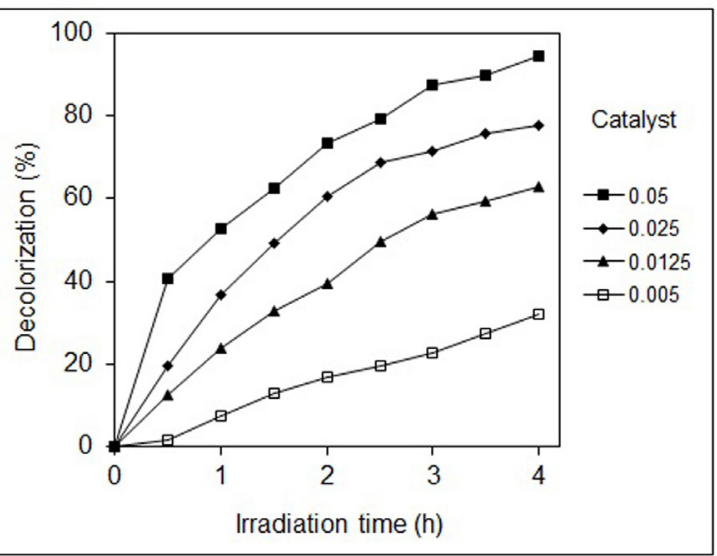

(b)

Fig. 6: AZCN dosage effect on degradation of dyes using UVIAZCN (a) BR18 and (b) BV16

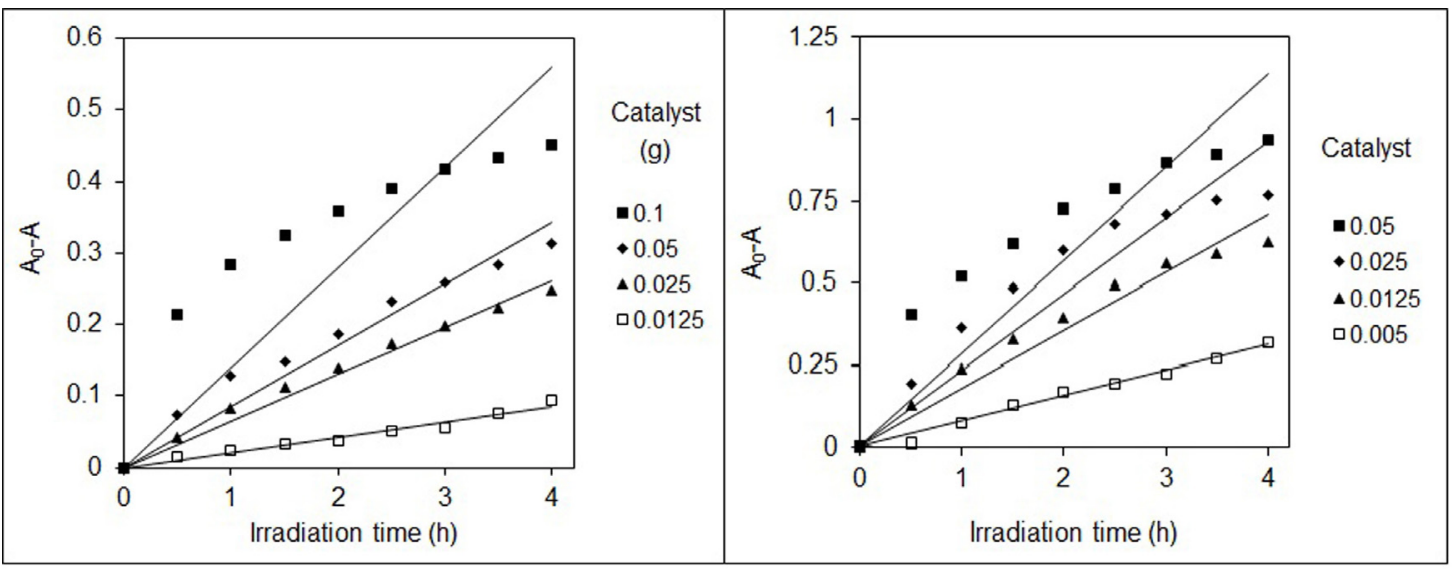

(a)

(b)

Fig. 7: The zero-order kinetic of photocatalytic dye degradation by AZCN at different catalyst dosages (a) BR18 and (b) BV16

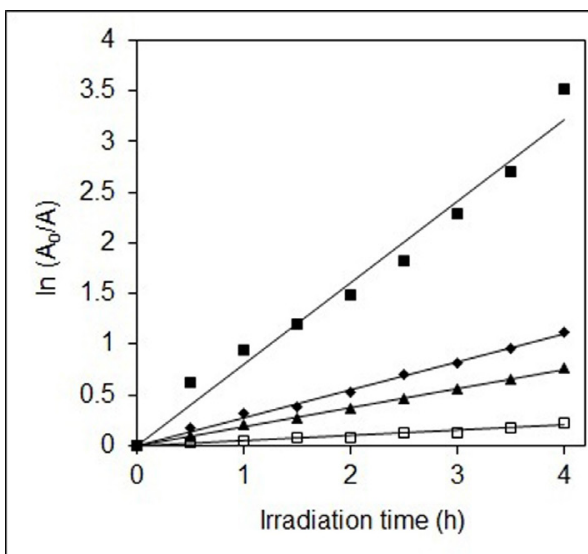

(a)

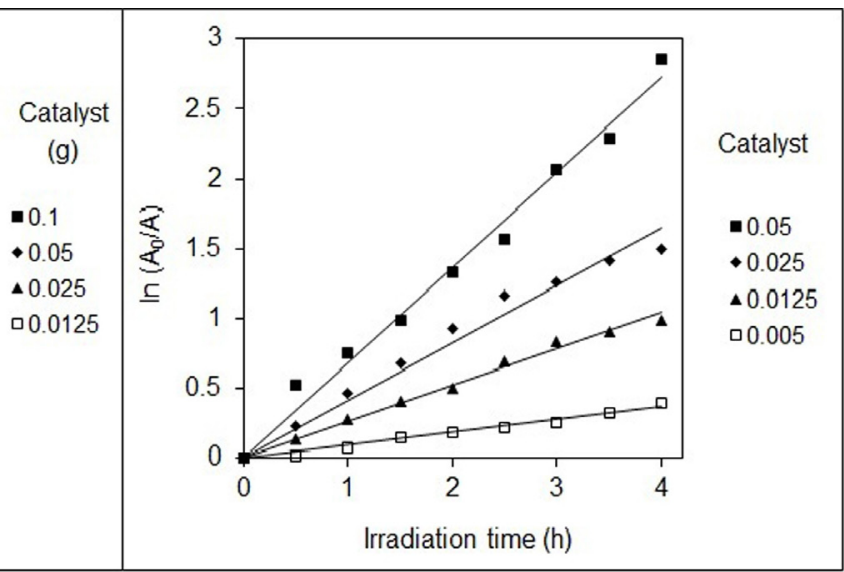

(b)

Fig. 8: The first-order kinetic of photocatalytic dye degradation by AZCN at different catalyst dosages (a) BR18 and (b) BV16 
surface. The $\mathrm{e}^{-/ \mathrm{h}^{+}}$pairs are then available for degradation processes, which usually involve the formation of reactive oxygen species, such as $\mathrm{HO}^{*}$ and $\mathrm{O}_{2}^{--}$, which then further aid in the degradation of pollutants.

The zero-order (eq. 1), first-order (eq. 2) and second-order (eq. 3) kinetics of photocatalytic dye degradation by the catalyst were studied (Table 1).

$$
\begin{aligned}
& A_{0}-A=k_{0} t \\
& \ln \left(A_{0} / A\right)=k_{1} t \\
& 1 / A=k_{2} t+1 / A_{0}
\end{aligned}
$$

where $A_{0}$ and $A$ are the initial dye absorbance and dye absorbance at time $t$, respectively. The $k_{0}, k_{1}$ and $k_{2}$ are the zero-order, first-order and second-order rate constants, respectively.

To introduce the applicability of the zero-order, first-order and second-order kinetics models for photocatalytic dye degradation by the nanocomposite at different catalyst dosages, linear plots of $A_{0}-A$ versus irradiation time (t) (Fig. 7), In $\left(A_{\delta} / A\right)$ versus irradiation time $(t)$ (Fig. 8 ) and $1 / A$ against irradiation time $(t)$ (Fig. 9) are plotted. The values of $k_{0}, k_{1}$ and $k_{2}, R^{2}$ (correlation coefficient values) are shown in Table 1.

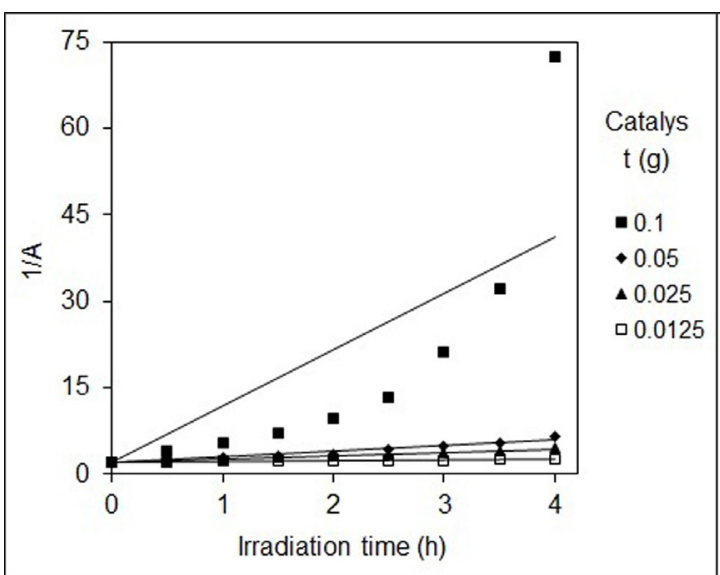

(a)

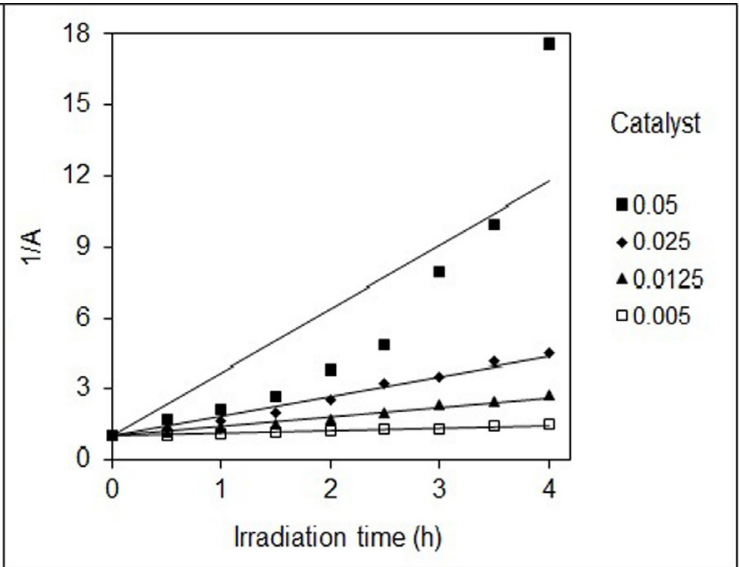

(b)

Fig. 9: The second-order kinetic of photocatalytic dye degradation by AZCN at different catalyst dosages (a) BR18 and (b) BV16

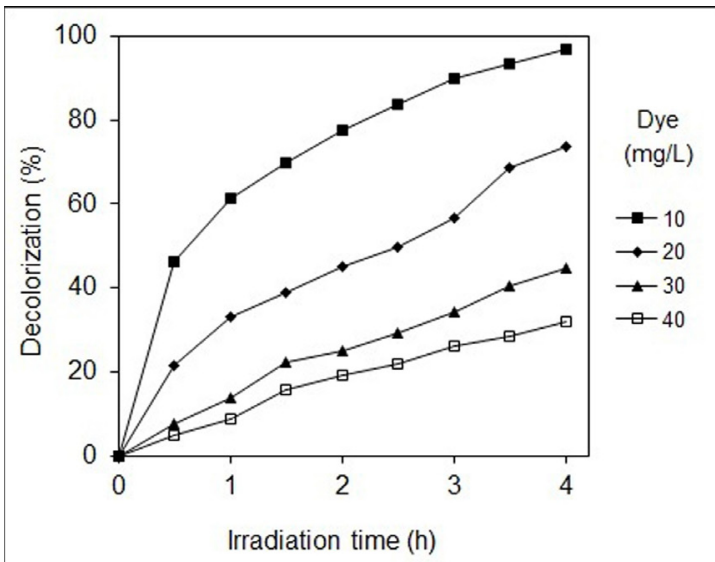

(a)

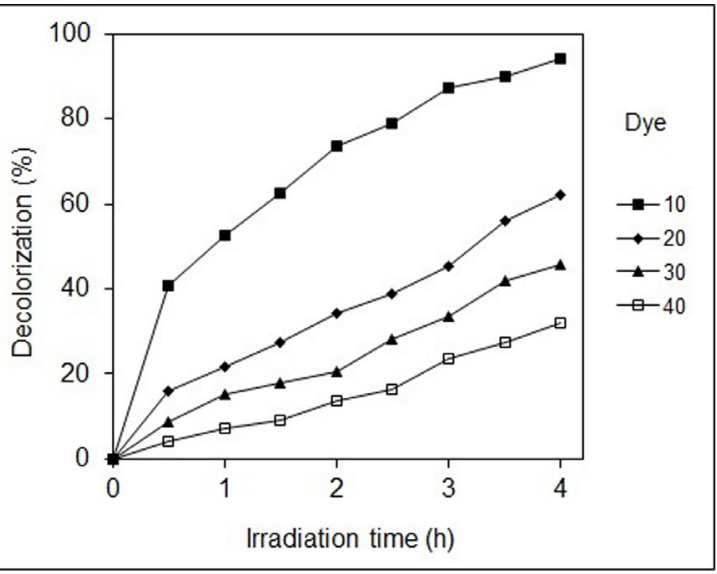

(b)

Fig. 10: Dye concentration effect on the degradation of dyes using UVIAZCN (a) BR18 and (b) BV16 
The results showed that the kinetics of photocatalytic dye degradation by AZCN at different catalyst dosages followed first-order kinetic model.

\section{Initial dye concentration}

Fig. 10 presents the effect of initial dye concentration on the photocatalytic dye degradation at different time intervals. The results showed that dye degradation decreases by initial dye concentration increasing. With the increase in the dye concentration, the possible cause is the interference from intermediates formed upon degradation of the parental dye molecules. Such suppression would be more pronounced in the presence of an elevated level of degradation intermediates formed upon an increased initial dye concentration ${ }^{12}$.

To introduce the applicability of the zero-order, first-order and second-order kinetics models for photocatalytic dye degradation by the nanocomposite at different initial dye concentrations, linear plots of $A_{o}-A$ versus irradiation time $(t)$ for zero-order model (Fig. 11), In ( $\left.A_{\delta} / A\right)$ versus irradiation time $(t)$ for first-order order model (Fig. 12) and $1 / A$ against irradiation time $(t)$ for second-order model (Fig. 13) are plotted. The values of $k_{0}, k_{1}$ and $k_{2}$, $R^{2}$ (correlation coefficient values) are shown in Table 2.

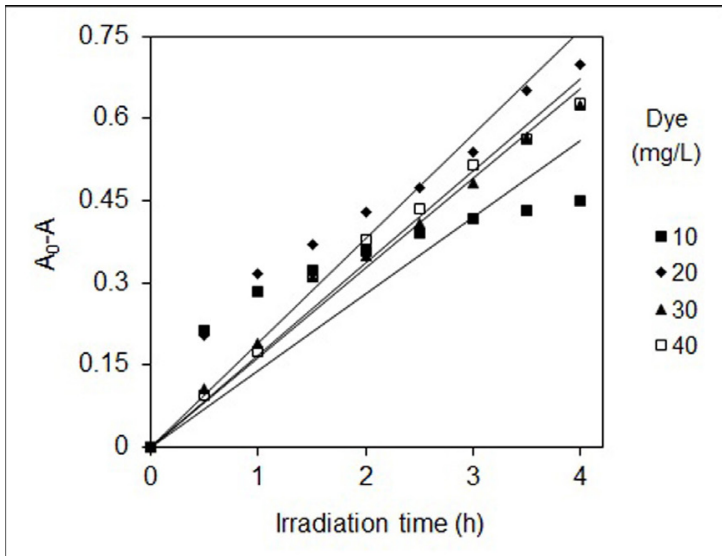

(a)

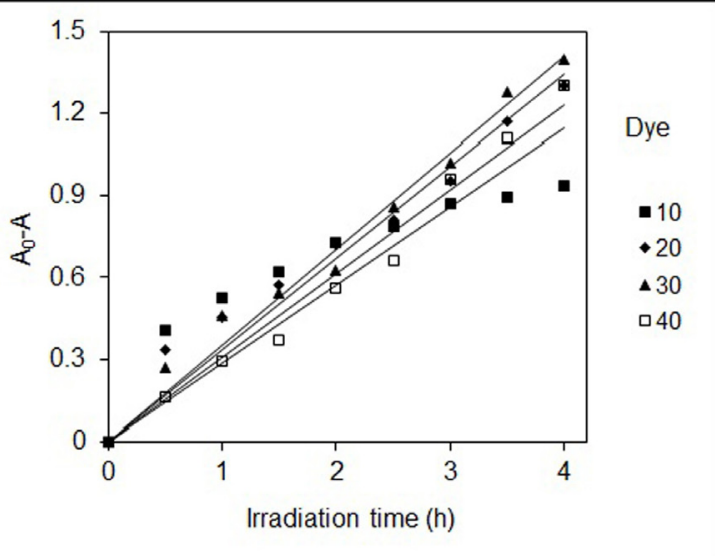

(b)

Fig. 11: The zero-order kinetic of photocatalytic dye degradation by AZCN at different dye concentrations (a) BR18 and (b) BV16

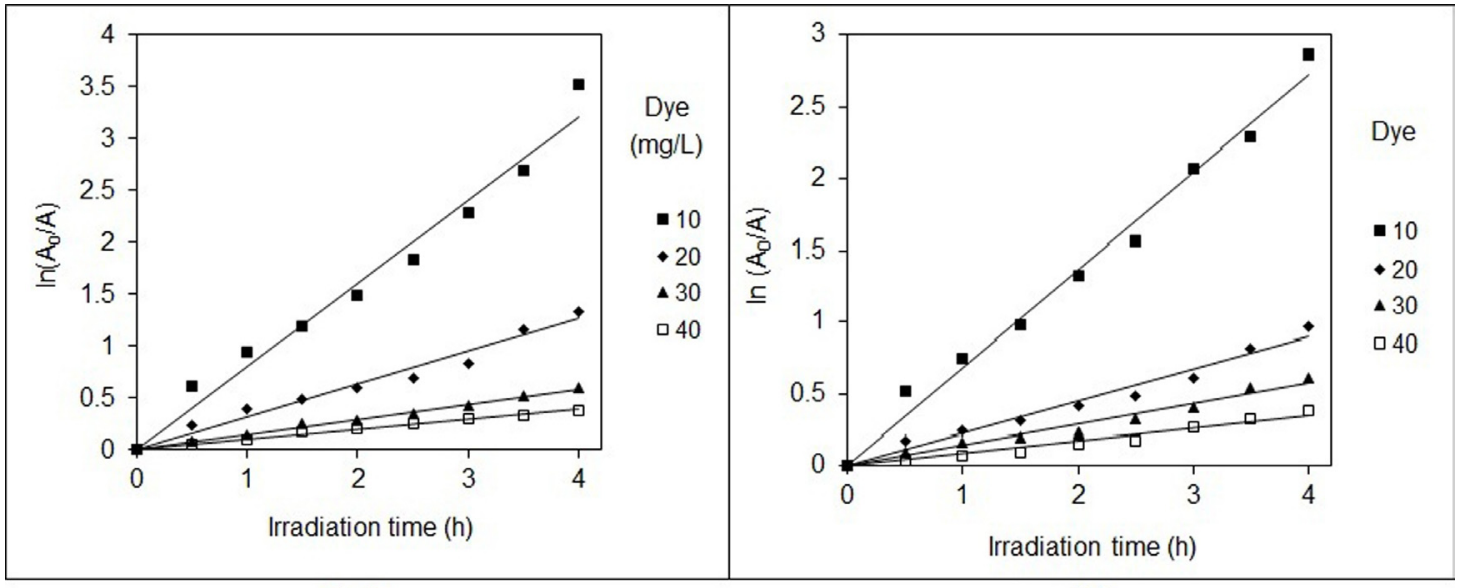

(a)

(b)

Fig. 12: The first-order kinetic of photocatalytic dye degradation by AZCN at different dye concentrations (a) BR18 and (b) BV16 
The results showed that the kinetics of photocatalytic dye degradation by AZCN at different initial dye concentrations followed first-order kinetic model.

\section{Salt}

Inorganic anions (salts) exist in wastewater. These materials may compete for the active sites on the AZCN surface or deactivate the AZCN and, subsequently, decrease the degradation rate of the target dyes. Hydroxyl radical is high reactive and nonselective radical. It reacts with non-target compounds present in the background water matrix such as dye auxiliaries in the dye bath. It causes higher $\mathrm{HO}$ ' demand to accomplish the desired degree of degradation, or complete inhibition of advanced degradation rate and efficiency ${ }^{16}$.

Fig. 14 shows the effect of salts on the photocatalytic dye degradation using AZCN. The anionic species could inhibit dye degradation through their scavenging effects on the $\mathrm{HO}^{\circ}$ radicals. To generate sufficient levels of $\mathrm{HO}^{*}$ for the photocatalytic dye degradation in the presence of high levels of sulfate, a higher catalyst dose should be used.

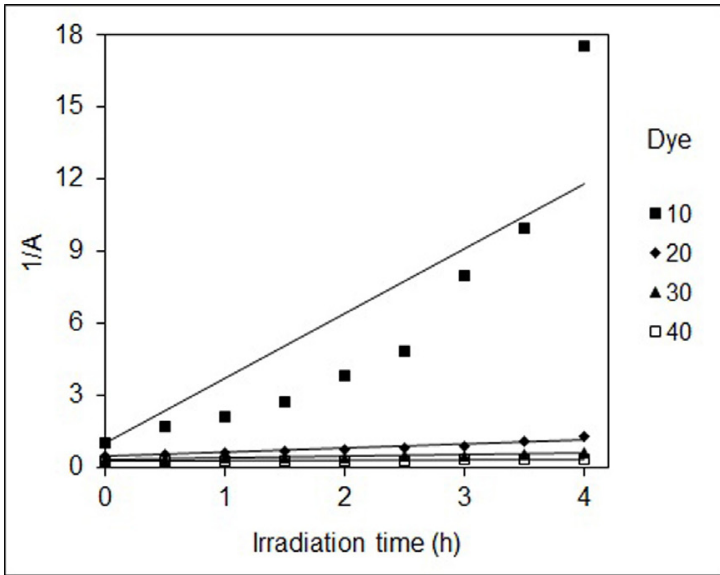

(a)

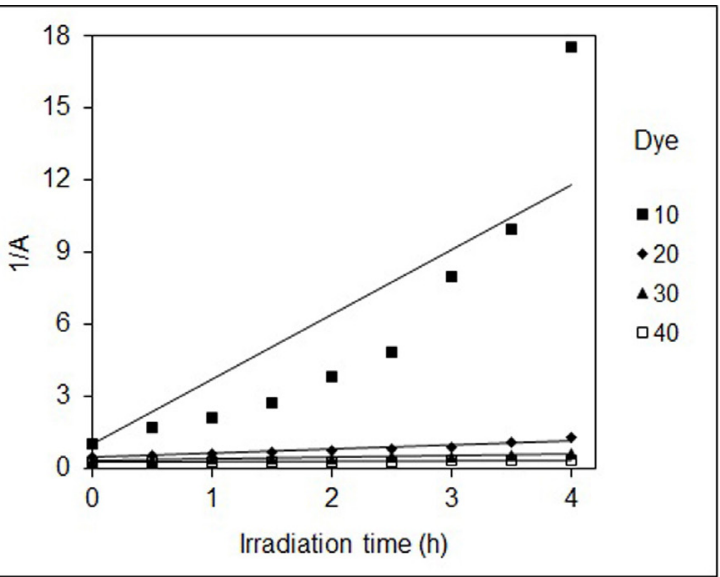

(b)

Fig. 13: The second-order kinetic of photocatalytic dye degradation by AZCN at different dye concentrations (a) BR18 and (b) BV16

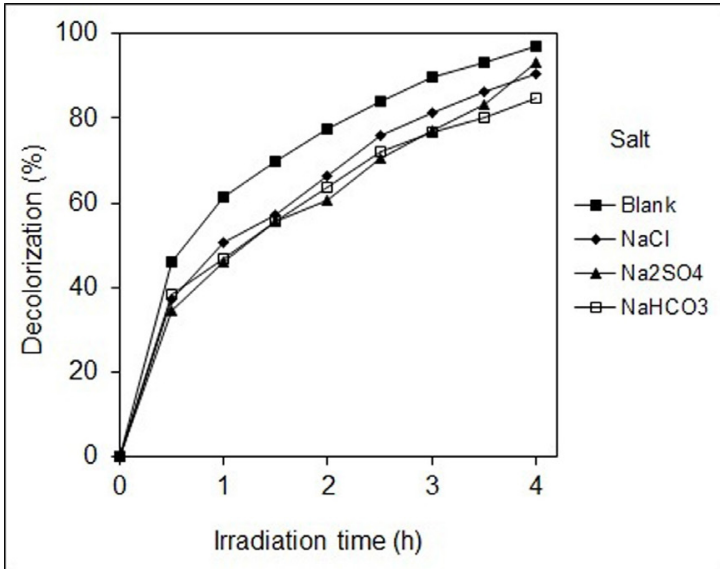

(a)

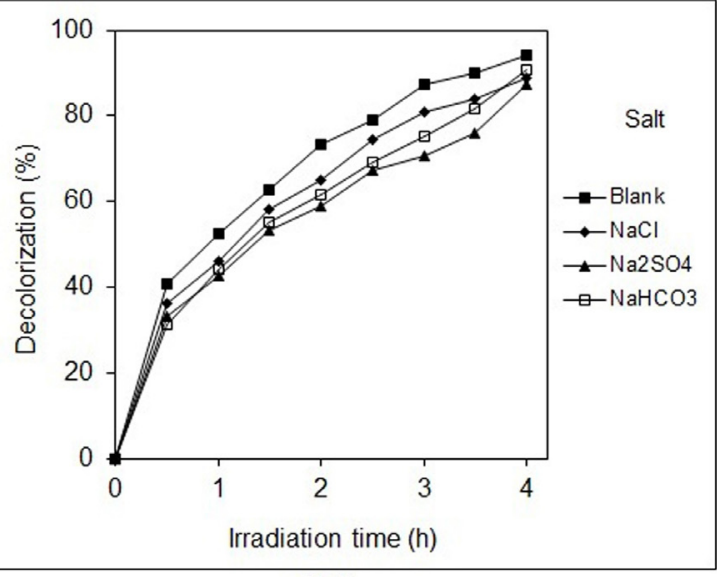

(b)

Fig. 14: Salt (inorganic anion) effect on degradation of dyes using UVIAZCN (a) BR18 and (b) BV16 
Table 1: The kinetics constants of photocatalytic dye degradation by $A Z C N$ at different catalyst dosages

\begin{tabular}{|c|c|c|c|c|c|c|}
\hline \multirow[t]{2}{*}{$A Z C N(g)$} & \multicolumn{2}{|c|}{ zero-order kinetic } & \multicolumn{2}{|c|}{ first-order kinetic } & \multicolumn{2}{|c|}{ second-order kinetic } \\
\hline & $\mathbf{k}_{0}$ & $\mathbf{R}^{2}$ & $\mathbf{k}_{1}$ & $\mathbf{R}^{2}$ & $\mathbf{k}_{2}$ & $\mathbf{R}^{2}$ \\
\hline & BR18 & & & & & \\
\hline 0.1000 & 0.1398 & 0.5158 & 0.8030 & 0.9756 & 9.7496 & 0.6079 \\
\hline 0.0500 & 0.0856 & 0.9438 & 0.2751 & 0.9944 & 0.9601 & 0.948 \\
\hline 0.0250 & 0.0655 & 0.9821 & 0.1869 & 0.9989 & 0.5539 & 0.9747 \\
\hline 0.0125 & $\begin{array}{c}0.0213 \\
\text { BV16 }\end{array}$ & 0.9312 & 0.0499 & 0.9599 & 0.1173 & 0.9446 \\
\hline 0.0500 & 0.2857 & 0.6558 & 0.6812 & 0.9874 & 2.7002 & 0.7428 \\
\hline 0.0250 & 0.2344 & 0.8267 & 0.4120 & 0.9746 & 0.8449 & 0.9605 \\
\hline 0.0125 & 0.1777 & 0.9425 & 0.2602 & 0.9931 & 0.4076 & 0.9789 \\
\hline 0.0050 & 0.0782 & 0.9692 & 0.0907 & 0.9847 & 0.1063 & 0.9707 \\
\hline
\end{tabular}

Table 2: The kinetics constants of photocatalytic dye degradation by AZCN at different dye concentrations

\begin{tabular}{lcccccc}
\hline $\begin{array}{l}\text { Dye } \\
(\mathbf{m g} / \mathbf{L})\end{array}$ & \multicolumn{2}{c}{ zero-order kinetic } & \multicolumn{2}{c}{ first-order kinetic } & \multicolumn{2}{c}{ second-order kinetic } \\
& $\mathbf{k}_{\mathbf{0}}$ & $\mathbf{R}^{\mathbf{2}}$ & $\mathbf{k}_{\mathbf{1}}$ & $\mathbf{R}^{\mathbf{2}}$ & $\mathbf{k}_{\mathbf{2}}$ & $\mathbf{R}^{\mathbf{2}}$ \\
\hline 10 & 0.1398 & 0.5158 & 0.803 & 0.9756 & 9.7496 & 0.6079 \\
20 & 0.1904 & 0.8918 & 0.3157 & 0.9681 & 0.5884 & 0.8801 \\
30 & 0.1638 & 0.9803 & 0.1459 & 0.9944 & 0.1329 & 0.9811 \\
40 & 0.1678 & 0.9773 & 0.0985 & 0.9917 & 0.0584 & 0.9963 \\
& $\mathrm{BV} 16$ & & & & & \\
10 & 0.2857 & 0.6558 & 0.6812 & 0.9874 & 2.7002 & 0.7428 \\
20 & 0.3352 & 0.9598 & 0.2245 & 0.9723 & 0.1600 & 0.9064 \\
30 & 0.3514 & 0.9828 & 0.1446 & 0.9740 & 0.0610 & 0.9384 \\
40 & 0.3079 & 0.9808 & 0.0875 & 0.9619 & 0.0251 & 0.9356 \\
\hline
\end{tabular}

\section{CONCLUSIONS}

In this paper, AZCN was synthesized and characterized. The photocatalytic dye degradation ability of AZCN from colored textile wastewater was studied. Reactive dyes were used as model compounds. The effects of AZCN dosage, initial dye concentration and salt on photocatalytic dye degradation were evaluated. The photodegradation with UV in the absence of AZCN presents a low performance for dye degradation from solutions $(\leq 5)$. The synergistic effects of AZCN and UV were observed because of the catalytically performance of $A Z C N$ for production of hydroxyl radicals. It can be concluded that the AZCN could be used as a photocatalyst to degrade dyes in wastewater.

\section{REFERENCES}

1. Malik, P. K. J. Haz. Mat. 2004, 113, 81-88.

2. Mahmoodi. N. M. J. Mol. Catal. A: Chem. 2013, 366, 254-260.

1. Yiçitoçlu, M; Temoçin, Z. Fibers and Polymers.
2010, 11, 996-1002.

2. Lee, H. C; Jeong, Y. G; Min, B. G; Lyoo, W. S; Lee, S. C. Fibers and Polymers. 2009, 10, 636-642. 
3. Bauer, C; Jacques, P; Kalt, A. J. Photochem. Photobiol., A. 2001, 140, 80-92.

4. Mahmoodi, N. M. Fibers and Polymers. 2014, 15, 273-280.

5. Erol, F; Ozbelge, T. A. Chem. Eng. J. 2008, 139, 272-285.

6. Hui, Q; Ye, J; Li, N; Xiong, J. Fibers and Polymers. 2014, 8, 1648-1655.

7. Zhang, P; Wang, Q; Zhang, J; Li, G; Wei, Q. Fibers and Polymers. 2014, 15, 30-34.

10. El Ghali, A; Baouab, M. H; Roudesli, M. S. Fibers and Polymers. 2013, 14, 65-75.

11. Merzouka, B; Madanib, K. H; Sekki, A. Using electrocoagulation-electroflotation technology to treat synthetic solution and textile wastewater, two case studies. Desalination. 2010, 250, 573-577.
12. Konstantinou, I. K; Albanis, T. A. Kinetic and Mechanistic Investigations. Applied Catalysis B: Environmental. 2004, 49, 1-14.

13. Mahmoodi, N. M; Abdi, J; Bastani, D. Journal of Environmental Health Science and Engineering. 2013, 48, 4255-4267.

14. Li, B. X; Wang, Y. F. Facile synthesis and photocatalytic activity of $\mathrm{ZnO}-\mathrm{CuO}$ nanocomposite. Superlattice Microst. 2010, 47, 615-623.

15. Pavia, D. L; Lampman, G. M; Kaiz, G. S. Introduction to Spectroscopy: A guide for Students of Organic Chemistry, WB. Saunders, Philadelphia (1987).

14. Alaton, I. A. Coloration Technol. 2003, 119, 345-353. 